\title{
Unmet Needs in the Treatment of Allergic Asthma: Potential Role of Novel Biologic Therapies
}

\author{
WILLIAM W. STORMS, MD
}

\begin{abstract}
OBJECTIVE: To provide a review of the current status of the treatment of asthma and introduce new and developing forms of therapy by means of a review of published literature on asthma and publications on new and emerging therapies. Increased public awareness of asthma, improved patient and provider education, implementation of national treatment guidelines, and availability of safe and effective therapies have combined to provide an effective response to the increase in asthma prevalence. However, the number of persons with poorly controlled asthma and asthma-related complications remains unacceptably high. This is particularly true for the relatively small cohort of patients with moderateto-severe asthma that is poorly controlled with inhaled corticosteroids and other standard-of-care medications. Consequently, these patients often experience frequent exacerbations, leading to a disproportionate consumption of asthma health care resources and a poor quality of life. The National Committee on Quality Assurance suggests that the negative impact of asthma can be minimized if health care providers implement aggressive asthma management programs that include patient education and appropriate medications. Newer therapies such as injectable anti-lgE may provide a benefit for many patients.
\end{abstract}

SUMMARY: Currently available asthma medications have been proven to be generally safe and effective for most asthma patients. However, the subset of patients with difficult-to-treat asthma who experience frequent exacerbations requiring emergency department visits or hospitalizations may benefit from novel therapies designed to target specific mechanisms underlying airway inflammation.

CONCLUSIONS: New therapies may help in the treatment of patients whose asthma is not controlled. These include anti-immunoglobulin $E$ ( $(\mathrm{gE})$ antibodies, cytokine modulators, and DNA vaccinations. Future research will determine if these targeted biologic therapies are a cost-effective means to improve the clinical and economic outcomes of asthma management.

KEYWORDS: Airway inflammation, Allergic asthma, Asthma management, Biologics, IgE blockers, Managed care

J Managed Care Pharm. 2003;9(6):534-43
A sthma is a chronic inflammatory disease of the airways characterized by episodic symptoms such as coughing, dyspnea, wheezing, chest tightness, variable obstruction of the tracheobronchial tree, and increased bronchial hyperresponsiveness to various stimuli. ${ }^{1}$ Several therapeutic advancements over the past decade have yielded a variety of safe and effective medications that allow the majority of patients with asthma to lead productive lives. In addition, comprehensive asthma treatment guidelines such as the National Asthma Education and Prevention Program (NAEPP) Expert Panel Report ${ }^{1}$ outlines the need for aggressive asthma management in order to improve both clinical and economic outcomes of the disease.

As a result of these efforts, the estimated number of persons with asthma attacks has declined slightly since 1997.2 However, a small subset of asthma patients, in particular those with moderateto-severe persistent disease that is difficult to treat and poorly controlled with current standard of care medications, remain at risk for frequent exacerbations, which require emergency department visits or hospitalizations to treat. In addition, these patients miss a substantial number of work or school days as a result of asthma and generally have a poor quality of life (QOL). ${ }^{2}$

Although difficult-to-treat patients represent less than 20\% of the asthma patient population, ${ }^{3}$ they consume a disproportionate share of asthma care resources. ${ }^{3-5}$ Consequently, these patients can potentially derive significant benefit from a simultaneous effort by health care practitioners, government agencies, and managed care plans to teach self-management techniques, improve adherence to prescribed therapy, increase compliance by health care professionals to national asthma treatment guidelines, and enhance QOL., ${ }^{6,7}$ In addition, these patients may be candidates for treatment with novel biologic agents designed to target specific steps in the inflammatory cascade that contribute to persistent airway inflammation.

\section{Epidemiology and Impact}

\section{Prevalence}

According to the Centers for Disease Control (CDC), the estimated annual prevalence of self-reported asthma increased from 31.4 per 1,000 population in 1980 to 55.6 per 1000 in $1995 .{ }^{2}$ In 1996, there was a slight decrease in the self-reported prevalence (to 54.6 per 1,000). This downward trend continued through 1999, with 38.4 persons per 1,000 reporting an episode of asthma or an asthma attack during the preceding 12 months. However, this trend may reflect a change in the methodology used by the CDC to measure asthma prevalence. Since 1997, the CDC includes only 
those patients with a medical diagnosis of asthma as opposed to an unconfirmed self-report of asthma. ${ }^{2}$ Despite this change, it appears as if the trend is toward a decreasing prevalence of asthma in the United States.

Clear estimates of the distribution of asthma severity among those diagnosed with asthma are not widely available. Fuhlbrigge et al. used a survey to identify the overall (global) impact of asthma. ${ }^{8}$ Global asthma burden was composed of short-term (1 month) symptom burden, long-term (12 months) symptom burden, and functional impact of asthma-related activity limitations. This study identified only $10.7 \%$ of individuals as having a global asthma burden consistent with mild intermittent disease and $77.3 \%$ with moderate-to-severe persistent disease. The most recent effort to classify asthma prevalence by disease severity was generated by the Asthma in America Survey conducted in 1998. ${ }^{9}$ For this survey, researchers interviewed 2,509 adults and children with asthma and classified their asthma severity using the symptom-based NAEPP severity classification scheme. As illustrated in Figure 1, the Asthma in America Survey results suggest that 39.0\% of patients are classified with mild intermittent asthma, 15.7\% with mild persistent, $22.1 \%$ with moderate persistent, and 19.1\% with severe persistent disease. ${ }^{9}$

\section{Unplanned Use of Medical Resources}

Although asthma in the majority of patients is well controlled, it appears as if up to $20 \%$ of asthma patients consume a disproportionate share of asthma health care resources. ${ }^{3}$ These patients frequently require unplanned medical attention and seek care in emergency departments (ED) and other urgent care facilities. From 1980 through 1999, the number of ED visits for asthma increased by $36 \%,{ }^{2}$ and in 2000 , there were 1.8 million ED visits for asthma (Figure $2^{10}$ ). The hospitalization rate for asthma peaked in the mid-1980s and has gradually declined since then²; in 2000, there were 465,000 asthma-related hospitalizations (Figure $3^{11}$ ).

\section{Mortality}

Although the number of deaths and death rates from asthma increased gradually from 1980 to 1995, it appears as if the mortality rates have started to plateau or decrease. ${ }^{2}$ In 1995, 5,637 deaths were attributed to asthma. This number fell to 4,487 by 2000. ${ }^{2}$ As with ED visits and hospitalizations, disparities exist with higher mortality rates documented among African Americans, women, and the elderly, with significant regional differences. ${ }^{2}$

Asthma mortality may be preventable in a significant number of patients. In 1996, the National Institutes of Health published the Working Group Report on the Quality of Asthma Care, which suggested that nearly $50 \%$ of asthma mortality is preventable and that asthma mortality is associated with inadequate or poor-quality medical care. ${ }^{12}$ Mortality rates, hospital admission rates, and ED utilization rates are greatest among low-income and minority populations. For example, in the New York City area, asthma hospitalization rates were found to be 3.6 times greater for children

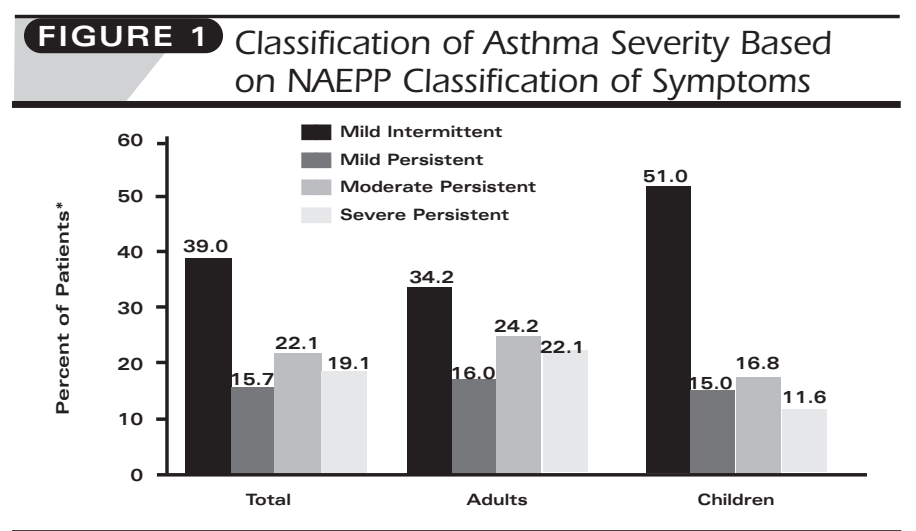

*Survey of 2,509 asthma patients: 1,788 Adults, 721 children.

NAEPP $=$ National Asthma Education and Prevention Program.

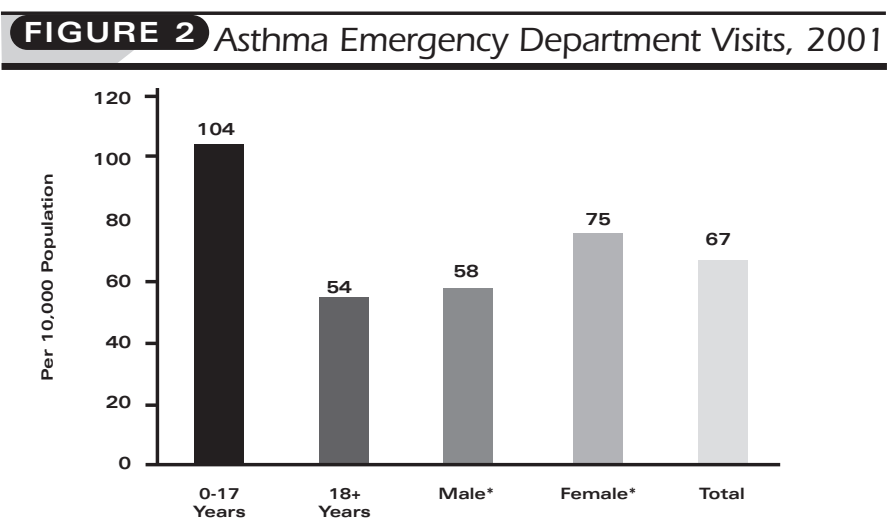

*Age adjusted to the 2000 population.

\section{FIGURE 3 Asthma Hospitalizations, 2001}

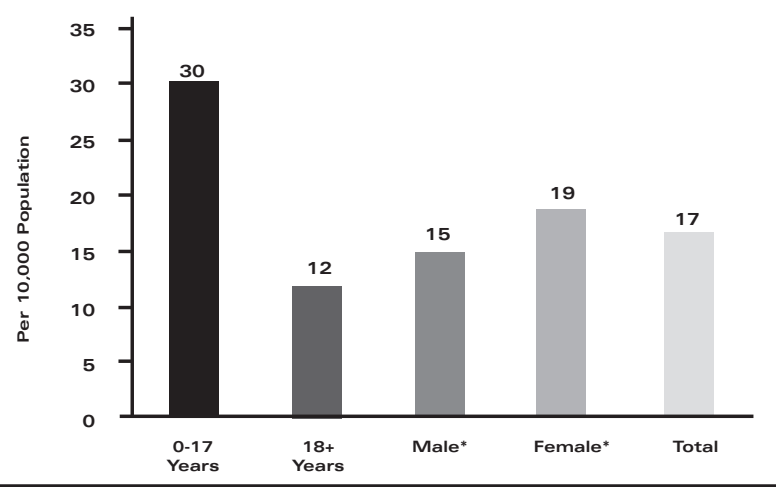

*Age adjusted to the 2000 population.

living in low-income areas than for those residing in higher-income areas. ${ }^{13}$ The difference noted in this survey may be attributed to an enhanced ability to identify, avoid, and control asthma triggers and better adherence to therapy in the higher-income groups. 


\section{Economic Burden}

In 2000, direct and indirect costs associated with asthma reached almost $\$ 12.7$ billion in the United States. ${ }^{14}$ Direct costs such as hospital and physician services, medications, and diagnostic tests account for $60 \%$, or $\$ 7.62$ billion, of the total amount spent on asthma care. ${ }^{14}$ Hospitalizations account for $47 \%$ of the direct medical expense, followed by medications (30\%), hospital outpatient visits (15\%), and ED visits (8\%). ${ }^{12}$ Indirect costs account for the remaining $\$ 5.08$ billion of the total expenditure and reflect the value of asthma-related absence from work, school, and other daily activities and loss of future potential earnings due to premature death. ${ }^{14}$ Morbidity costs are the largest component of indirect costs, contributing up to $75 \%$ of the total indirect costs for asthma. ${ }^{15,16}$ Loss of QOL also adds to the indirect costs; patients with the lowest scores on a health-related QOL scale have been shown to have the highest asthma-related health care utilization and cost. ${ }^{17}$

\section{Asthma Severity and Health Care Resource Utilization}

A close relationship exists between the severity of asthma and the costs associated with the disease. The volume of health care resources utilized and the number of lost days of school or work all increase as the severity of asthma increases. ${ }^{3-5}$ In particular, estimated costs associated with moderate disease are nearly twice those of mild asthma, and health care expenditures associated with severe asthma are more than 6 times those of mild asthma. ${ }^{4}$

In one study, patients considered "high cost" required a greater number of ED visits, hospitalizations, medications, and office/clinic visits; $80 \%$ of asthma-related health care resources were used by $20 \%$ of the population. ${ }^{5}$ High users of health care resources also have the highest risk of asthma-related morbidity and mortality. ${ }^{15,16}$ It is believed that high users of health care resources have persistent airway inflammation that is poorly controlled by current standard-of-care medications, suggesting that either these medications are not taken, are taken incorrectly, or are ineffective. ${ }^{18}$

A recent review of a database of privately insured individuals indicated that approximately $15 \%$ of asthma patients experience a moderate-to-severe exacerbation annually. The mean total annual direct costs associated with the cohort of patients with diagnoses of moderate-to-severe exacerbations (15\% of overall asthma patients) was estimated to be $\$ 10,900$. Direct costs are influenced by asthma-related and nonasthma-related health care utilization. Additionally, asthma patients who experience serious exacerbations tend to have more comorbidities, contributing to higher rates of hospitalization and ED visits compared with patients who do not have a diagnosis related to a moderate-to-severe exacerbation. ${ }^{19}$

Findings from the Epidemiology and Natural History of Asthma: Outcomes and Treatment Regimens (TENOR) study in severe and difficult-to-treat asthma confirm the observation that patients with inadequately controlled moderate-to-severe disease consumed a disproportionate share of health care resources. TENOR enrollees, a 3-year observational study of more than 4,500 patients with moderate-to-severe and/or difficult-to-treat disease, experienced more asthma-related hospitalizations and ED visits and had significantly lower asthma-related QOL compared with those with less-severe disease..$^{20}$ Additionally, TENOR patients who used multiple medications reported more problems with asthma control, higher rates of asthma-related hospitalizations and ED visits, and lower QOL compared with those who took fewer controller medications. ${ }^{20}$

\section{Asthma and Quality of Life}

In general, patients with asthma experience decreased classroom and workplace productivity, ${ }^{21,22}$ social maladjustment, ${ }^{23}$ and sexual dysfunction. ${ }^{24}$ Impairment in the health-related quality of life (HRQOL) in asthma patients has been demonstrated to contribute to rates of health care utilization. Eisner et al. ${ }^{17}$ conducted a random sample of 3,842 members of a staff model health maintenance organization (HMO) who completed an HRQOL survey. Patients were stratified for disease severity. The results of the survey indicated that better baseline asthma-specific HRQOL scores were associated with lower risk of asthma-related ED use or hospitalization. More favorable HRQOL scores were also associated with decreased asthma-related health care costs during the following year. ${ }^{17}$

Impaired QOL can also have a substantial impact on attendance and productivity at work and school. CDC data published in 2002 reveals that school absence days among children increased from 6.6 million in 1980 to 14.6 million in 1992. However this number dipped to 14 million in 1996. Among adults with asthma, work absence days increased from 6.2 million in 1982 to 14.5 million in $1996 .^{2}$

\section{Trends in the Treatment of Asthma}

\section{Undertreatment of Asthma}

To favorably improve both clinical and economic outcomes, an asthma management strategy must include accurate diagnosis, appropriate classification of disease severity, and therapeutic interventions designed to minimize or prevent the underlying airway inflammation. According to the 2001 report of The National Committee on Quality Assurance (NCQA ${ }^{7}$ ), approximately two thirds of patients enrolled in nongovernment managed care organizations were being treated with the appropriate asthma medications, representing an approximately $4 \%$ increase across all age groups over the previous year. With continued implementation of aggressive management strategies, this number will continue to rise and thus improve clinical and economic outcomes and enhance QOL. This is particularly critical for patients who fail to receive appropriate asthma care.

Several factors have been identified that may contribute to the undertreatment of asthma, including poor compliance with national asthma guidelines by physicians, pharmacists, and other caregivers, ${ }^{25}$ poor adherence by patients to prescribed therapeutic regimens, ${ }^{26-31}$ confusion regarding diagnosis due to the presence of comorbid conditions, ${ }^{32,33}$ unidentified exacer- 
bating factors or triggers, ${ }^{34}$ and medical or social conditions (e.g., depression, anxiety disorders, mental impairments, etc.) that limit the ability of the patient to understand the importance of adhering to prescribed therapies. ${ }^{35,36}$

\section{Effect of Medical Guidelines}

Evidence-based medicine is a movement away from reliance on professional judgment toward a more structured assessment of clinical knowledge. This approach provides a method of weighing health effects, economic impact, and patient preferences. The validity of clinical findings in the literature, including the effectiveness, applicability, and potential adverse effects of interventions, can be critically assessed and then evaluated by use of this decisionmaking tool. The majority of the recommendations in the current NAEPP asthma treatment guidelines are based on an evidencebased analysis of the literature. For example, the recommendation that inhaled corticosteriods (ICSs) form the basis of long-term asthma management is derived from an analysis that revealed that longterm regular use of these agents reduced asthma mortality. ${ }^{37}$

The first Expert Panel Report on the Management of Asthma was published in 1991 and recognized the role of airway inflammation in the pathogenesis of asthma. Subsequent editions of the Expert Panel Report were published in $1997^{1}$ and 2002.38 Although the NAEPP strives to keep clinical practice guidelines up-to-date, the continual emergence of new data on medications, asthma-monitoring techniques, and prevention programs makes this task challenging. For example, in 2002, the NAEPP Expert Panel published an extensive review of data published since 1997 on the long-term use of ICSs in children with asthma. Data describing the effect of combination therapy and use of antibiotics in the treatment of asthma as well as the impact of written asthma action plans and prevention programs are also reviewed..$^{38}$

Much progress has been made in the implementation of the recommendations provided in the NAEPP guidelines, but deviations from the guidelines in the care of asthma has been observed in several patient groups, including pediatric, inner-city, and managed care patient populations. ${ }^{39-42}$ Donahue et al. analyzed 19961997 claims data to compare baseline pharmacotherapy in children with asthma at 3 managed care organizations. ${ }^{40}$ Of the 13,352 children included in the study, fewer than than $40 \%$ were given controller medications during the study interval, leading the investigators to conclude that the pattern of asthma therapy in this pediatric population does not reflect the recommendations of the guidelines. ${ }^{40}$ Lang et al. performed a cross-sectional analysis of monthly bronchodilator and ICS prescription rates and demographic factors such as race, ethnicity, poverty, educational levels, and ZIP code and noted the existence of a gap between optimal asthma drug prescribing as recommended by the guidelines and actual prescribing patterns. ${ }^{41}$ This gap was greatest in asthma patients living in the ZIP codes assigned to inner-city regions of Philadelphia. Legorreta et al. surveyed 5,580 members of a large California HMO to compare the status of asthma disease manage- ment with treatment recommendations found in the NAEPP guidelines. ${ }^{42}$ These authors reported that $72 \%$ of survey respondents reported having a steroid inhaler, but only $54 \%$ used it daily. Similarly, 26\% reported having a peak-flow meter, but only 16\% used it daily. ${ }^{42}$ Doerschug et al. reported that practitioner training and specialization significantly influenced understanding and subsequent compliance with the NAEPP guidelines. ${ }^{39}$ These investigators observed that asthma specialists as well as generalists with more advanced training in asthma had a greater understanding of the guidelines. ${ }^{39}$

Additional barriers to compliance with practice guidelines in general include disagreement with the recommendations, ${ }^{43-45}$ lack of familiarity and training, ${ }^{43-45}$ economic disincentives ${ }^{46}$ and inadequate time. ${ }^{47}$ These results suggest a need for more and better educational programs that address asthma diagnosis and treatment targeted to health care professionals and patients regarding the importance of national guidelines.

\section{Impact of Managed Care Intervention}

Managed care organizations have contributed to the improvement observed in asthma outcomes via the initiation of asthma management programs that identify high-risk patients, motivate providers to comply with national treatment guidelines, educate patients about their disease, and monitor clinical and economic outcomes. One of the most successful programs implemented by managed care plans uses comprehensive asthma management clinics that enlist the support of pharmacists, primary care physicians, asthma specialists, and case managers. Specifically, pharmacist-managed asthma care programs have been demonstrated to improve compliance with treatment guidelines, increase patient understanding of the disease and adherence to therapy, enhance QOL, and improve clinical and economic outcomes.

A pharmacist-provided comprehensive education program in conjunction with care provided by a pulmonologist improved the economic, clinical, and humanistic outcomes in adults with asthma compared with patients receiving care from a pulmonologist alone. ${ }^{48}$ Patients receiving combined care reported significantly more information about asthma self-management, were more likely to monitor peak-flow readings, and had increased satisfaction with care than those treated exclusively by the specialist. Fischer et al. reported that pharmacist intervention at the point of sale appeared to increase the information given to patients about their medications, increase awareness of side effects, and promote adherence compared with usual care. ${ }^{49}$

Narhi et al. conducted a 12-month prospective study to determine the impact of community pharmacist intervention on severity of asthma symptoms, changes in peak-flow rates, changes in daily medications, and the number of patients requiring oral corticosteroids. ${ }^{50}$ A positive change was noted in all outcome measures, particularly in the severity of asthma symptoms, with $79 \%$ of patients having a net improvement in one or more indicators of asthma severity. ${ }^{50}$ 
A drug therapy monitoring clinic established by the pharmacy department of a large military hospital was highly effective in improving clinical and economic outcomes for asthma patients. ${ }^{51}$ A chart review following implementation of the program indicated that compliance with national treatment guidelines was significantly improved when patients were followed by a pharmacist compared with a primary care physician..$^{51}$

A recently reported randomized controlled clinical trial indicates that 12 months after implementation of an aggressive pharmacist-managed intervention program, asthma patients demonstrated increased peak-flow rates and satisfaction with care compared with usual care, but they also increased the amount of breathing-related medical care sought. Consequently, the program increased the overall cost of care. However, disease severity and patient awareness of available treatment options may explain the apparent lack of cost savings with this intervention. ${ }^{52}$

Referral to an asthma specialist (usually an allergist or pulmonologist) — particularly for patients with severe asthma — may help improve medical adherence to asthma management guidelines and patient QOL. ${ }^{53-55}$ Westley et al. $^{56}$ reviewed the charts of 70 moderate-to-severe asthma patients enrolled in a large staff model HMO before and after referral to an asthma specialist (either allergist or pulmonologist). Following consultation with the specialist, there was a $45 \%$ decrease in the number of office visits for asthma, a 55\% decrease in acute care visits, a 67\% decrease in hospitalizations, and a cost savings of $\$ 2,100$ per patient. ${ }^{56}$

Similar findings were reported by Wu et al., ${ }^{57}$ who conducted a survey of asthma outcomes among 1954 adult asthma patients treated either by a specialist or generalist. Compared with patients treated by generalists, patients treated by an allergist reported fewer cancelled activities, hospitalizations, and ED visits and greater physical functioning. Patients treated by pulmonologists reported that their symptom control was improved compared with those treated by generalists. ${ }^{57}$ Frieri et al. ${ }^{58}$ reported that asthma patients evaluated by allergists had more severe disease and thus had more office visits and were prescribed more medications than those seen by a primary care physician. However, it was also noted that, despite the increased cost to treat the more severe patients, their care was more closely aligned with the NAEPP treatment guidelines.

\section{Adherence to Asthma Therapy}

Patients who adhere closely to therapy tend to do well clinically.59 Conversely, poor adherence to a therapeutic regimen has been identified as a major factor contributing to suboptimal asthma control. ${ }^{60-62}$ Nonadherence exacts multiple consequences, including increased hospitalization, ED visits, detrimental changes in clinical status, and asthma-related mortality. ${ }^{63-65}$ For example, Suissa et al. have reported the hospitalization rate and incidence of asthmarelated death are lower in patients who persist with continuous low-dose asthma therapy compared with those who do not. ${ }^{66,67}$

Adherence rates for all medications prescribed for asthma ther- apy range from 30\% to $70 \% .{ }^{64}$ In several reports, fewer than half of all patients prescribed inhaled medications adhered to their prescribed regimens. ${ }^{65,68}$ Adherence to ICS therapy has been reported to decrease by $1.6 \%$ a week, ${ }^{62}$ possibly because patients perceive ICS therapy to be time-consuming, inconvenient, and difficult to use. $^{62}$ Alternatively, the reduction in patient adherence may be explained by the efficacy of current treatments such as ICS therapy as patients become complacent in the absence of recurrent asthma symptoms.

\section{Strategies to Improve Adherence}

There is no single reason for poor adherence to therapy, and nonadherence can occur with both symptomatic and asymptomatic diseases. However, adherence is a learned behavior and can be improved with practice and reinforcement. Therefore, it appears that some form of ongoing intervention from pharmacists or other health care practitioners is imperative to increase adherence to prescribed treatment regimens. Strategies should include methods that both health care professionals and patients can implement.

Educating patients is a cost-effective way to improve asthma outcomes, particularly among high-risk patients. ${ }^{69,70}$ Patients who are knowledgeable about their disease and the treatments being used to manage it are also more motivated to adhere to the treatment plan. ${ }^{71}$ An educational dialogue founded on open communication between clinician and patient is critical for a successful partnership in asthma care. ${ }^{61}$

Educating patients about asthma self-management begins at the time of diagnosis and is integrated into each clinician-patient interaction. All patients with moderate-to-severe asthma should be enrolled in an ongoing, intensive asthma education program. Primary caregivers should be enrolled for those whose care must be delegated to others. Patients should receive instruction on the appropriate use of medication delivery devices and should be able to demonstrate their self-administration technique to the satisfaction of their health care provider. In addition, patients should be educated about asthma pathophysiology, environmental control and avoidance measures, asthma action plans, medications, drug interactions and side effects, self-management, and adherence techniques. Educational mediums include discussions with health care professionals, brochures, videos, and information acquired via the Internet or support groups.

As part of the educational effort, each patient should be given a written asthma "action plan" that outlines the asthma management program design in partnership with the patient and family. ${ }^{72}$ A sound action plan is easy to understand and implement, consistent with the patient's personal goals and daily activities, and outlines how variations in symptoms impact dosing of medication. Specifically, the asthma action plan includes a diary for recording peak expiratory flow-rate measurements and therapeutic guidelines to follow when peak-flow measurements decline or symptoms worsen. The plan should also clearly outline when it is appropriate to seek emergency help. Pertinent phone numbers 
(clinic, hospital, pharmacy, urgent care center, etc.) should be clearly written in a prominent place in the action plan. An excellent example of an asthma action plan is found in the NAEPP guidelines. ${ }^{1,38}$

\section{Biologic Therapies for the Treatment of Asthma}

Pharmacologic therapy is used to prevent and control asthma symptoms, reduce frequency and severity of exacerbations, and reverse airflow obstructions. Choosing the best medication for individual patients requires knowledge of the severity of the airway inflammation, familiarity with the mechanism of action of the drug(s), the likelihood of patient adherence, and expected impact on QOL and economic outcomes resulting from the use of each medication.

Current therapies are safe and effective for the vast majority of asthma patients and lead to improved clinical and economic outcomes. However, as noted earlier, a relatively small proportion of asthma is poorly controlled on current therapies. It is these patients who may benefit from therapies developed as a result of recent advances in the understanding of the pathogenesis of asthma.

The novel therapies reviewed briefly below have the potential to inhibit the allergic inflammatory process or modify the natural history of the asthma, although many of these benefits have yet to be proven beyond small clinical studies. An anti-immunoglobulin E (IgE) antibody has recently been approved for use in patients with moderate-to-severe asthma poorly controlled on ICS therapy. Therapies under development, but not currently available outside of clinical trials, include cytokine modulators, immunostimulatory DNA sequences, and monoclonal antibodies targeted against Th2 cytokines and mediators.

\section{Anti-lgE Antibodies}

The anti-IgE antibody (or IgE blocker) omalizumab is the first biologic therapy to be approved by the U.S. Food and Drug Administration (FDA) for the treatment of allergic inflammation in asthma. Specific FDA approval was granted for adults and adolescents aged 12 years and older with moderate-to-severe persistent asthma who have a positive skin test or in vitro reactivity to a perennial aeroallergen and whose symptoms are inadequately controlled with ICSs. ${ }^{73}$

IgE blockers inhibit binding of free IgE to receptors on the proinflammatory mast cells and basophils, thus attenuating the cascade of events that leads to airway inflammation and subsequent symptoms in patients with moderate-to-severe allergic asthma. 1,38 Once- or twice-monthly subcutaneous administration of omalizumab to patients who remained symptomatic on a constant dose of ICSs resulted in a significant $(P \leq 0.005)$ reduction in the frequency of exacerbations compared with ICS therapy alone (Table $1^{74,75}$ ). An exacerbation was defined as an episode severe enough to require either a doubling of baseline ICS dose or addition of a course of systemic corticosteroids, based on the treating physician's clinical judgment. The addition of omalizumab to ICS

\section{TABLE 1 Placebo-Controlled Trials of Omalizumab in Moderate-to-Severe Asthma: Frequency of Exacerbations}

\begin{tabular}{|c|c|c|c|c|}
\hline & \multicolumn{2}{|c|}{ Busse et. al $^{74}$} & \multicolumn{2}{|l|}{ Soler et. $\mathbf{a l}^{75}$} \\
\hline & $\begin{array}{c}\text { Omalizumab } \\
(\mathrm{N}=268)\end{array}$ & \begin{tabular}{|l|} 
Placebo \\
$(\mathrm{N}=257)$
\end{tabular} & $\underset{(N=274)}{\text { Omalizumab }}$ & $\begin{array}{l}\text { Placebo } \\
(\mathrm{N}=272)\end{array}$ \\
\hline & \multicolumn{4}{|c|}{ Stable Steroid Phase (16 weeks) } \\
\hline $\begin{array}{c}\text { Mean no. exacerbations } \\
\text { per patient }\end{array}$ & 0.28 & 0.54 & 0.28 & 0.66 \\
\hline \multirow[t]{2}{*}{$P$ Value } & \multicolumn{2}{|l|}{0.009} & \multicolumn{2}{|l|}{$<0.001$} \\
\hline & \multicolumn{4}{|c|}{ Steroid Reduction Phase (12 weeks) } \\
\hline $\begin{array}{c}\text { Mean no. exacerbations } \\
\text { per patient }\end{array}$ & 0.39 & 0.66 & 0.36 & 0.75 \\
\hline$P$ Value & \multicolumn{2}{|l|}{0.03} & \multicolumn{2}{|c|}{$<0.001$} \\
\hline
\end{tabular}

therapy also increased forced expiratory volume in 1 second $\left(\mathrm{FEV}_{1}\right)$ from baseline $(P<0.05)$ and improved daytime and nocturnal asthma symptom scores compared with the control $(P<0.05){ }^{74,75}$

Anaphylaxis or an anaphylactoid reaction has been reported within 2 hours of administration of omalizumab in $<0.1 \%$ of all patients receiving omalizumab without other identifiable allergic triggers. No adverse drug interactions or antibodies to omalizumab have been reported. Among all completed studies, malignant neoplasms (excluding nonmelanoma skin cancers) occurred in 16 of 4,127 patients exposed to the drug and 2 of 2,236 controls. The most frequent adverse events included injection site reaction (45\%), viral infections (23\%), upper respiratory tract infection (20\%), sinusitis (16\%), headache (15\%), and pharyngitis (11\%). These events were observed at similar rates in omalizumab-treated patients and control patients..$^{73}$

Due to the need for subcutaneous administration, cost, and narrow indication, biologic therapies such as omalizumab, while promising, are not currently recommended for use in large numbers of asthma patients. Rather, their use should be targeted to the approximately $30 \%$ of asthma patients with a documented allergic component that experience frequent exacerbations, have a history of high health care resource utilization, have a poor record of adherence to therapy, and in whom therapy may be complicated by IgE-mediated comorbidities. This new drug therapy is costly compared with existing therapies but may be cost effective if targeted to the most appropriate patients. A thorough description of the cost-effectiveness of omalizumab awaits further investigation.

\section{Agents Targeting Cytokine Activity}

The effects of key inflammatory cytokines can be attenuated by several experimental monoclonal antibodies currently in development. These antibodies have their effects by blocking the cytokine receptor or by binding to the cytokine in a way that renders them inactivate. ${ }^{76}$ Soluble IL-4 (interleukin) receptor antagonists (IL-4R) are reported to improve lung function and reduce corticosteroid and $\mathrm{B}_{2}$-agonist use in moderate asthma, possibly via a mechanism that attenuates the production of $\operatorname{IgE}$ and expression 
of IgE receptors. ${ }^{77,78}$

Initial phase 1 and phase 2 studies with soluble IL-4R were performed in subjects with mild-to-moderate persistent asthma who were withdrawn from ICS therapy and then randomly assigned to placebo or a single dose of nebulized IL-4R. ${ }^{76,78}$ Nebulized IL-4R therapy improved asthma symptom scores, decreased rescue $\mathrm{B}_{2}$-agonist use, improved $\mathrm{FEV}_{1}$, and decreased methacholine airway responsiveness. No significant side effects were noted. A subsequent phase 2 study in subjects with moderate persistent asthma compared the longer-term effect of administering weekly doses of nebulized IL-4R for 3 months as opposed to a single dose. Withdrawal of ICSs in subjects treated with placebo resulted in a significant decline in $\mathrm{FEV}_{1}$ that did not occur in the treatment group. Although much work remains, these studies suggest the potential of IL-4R therapy in asthma.

Initial studies with anti-IL-5 were performed in patients with mild asthma exposed to an inhaled allergen to determine if anti-IL-5 provided protection against eosinophilia and airway responsiveness to methacholine. ${ }^{79} \mathrm{~A}$ single intravenous infusion of anti-IL-5 antibody reduced blood and sputum levels of eosinophils by more than $90 \%$. However, despite this reduction, patients treated with anti-IL-5 were not protected against the latephase decline in FEV 1 and did not develop reduced airway responsiveness. ${ }^{79}$

A second study demonstrated that while anti-IL-5 is very effective in inhibiting eosinophils in blood and sputum (i.e., >90\%), it is less effective in inhibiting airway eosinophils (only 60\%). This level of eosinohil reduction may be insufficient to attenuate or prevent airway hyperreactivity.

Studies conducted in IL-5 and eotaxin-deficient mice demonstrated that eosinophil recruitment to the airway and development of airway hyperreactivity is maximally inhibited in the combined IL-5/eotaxin-deficient mice as opposed to the individual mutant mice. These results suggest that the combination of anti-IL-5 with an inhibitor of eosinophil chemoattraction may be a more effective strategy than inhibiting IL-5 or eotaxin alone. ${ }^{80}$

Another approach to altering cytokine activity includes the administration of anti-inflammatory cytokines. IL-10 has several anti-inflammatory properties that may make it important in the treatment of asthma. Reduced levels of IL-10 have been noted in asthma patients, and patients with severe asthma are more likely to exhibit gene expression associated with lower production of IL-10. ${ }^{78,81}$ However, some studies have demonstrated increased IL-10 mRNA (messenger RNA) expression in allergy and asthma. ${ }^{82}$ Clearly, additional research is required to determine the role of IL-10 in asthma and its potential as a therapy for asthma.

The cytokine IL-12 plays a role in regulating Thl cell differentiation and in blocking the expansion of Th2 cell lines by stimulating Th0 cells to release IFN-g (interferon). ${ }^{77}$ There are reports that recombinant IL-12 reduces eosinophils in blood and sputum but has little or no effect on bronchial hyperresponsiveness. ${ }^{83}$ Although these experimental therapies hold some promise, it is believed that modifying individual cytokines may not ultimately prove to be an effective strategy because of the significant redundancy in the immune response to inhaled allergens. ${ }^{76} \mathrm{~A}$ novel soluble receptor for IL-13 may also have therapeutic potential for the treatment of asthma. ${ }^{76}$

The cytokine modulator suplatast tosilate is a nonspecific Th2 inhibitor that has been shown to suppress IL-4 and IL-5 synthesis and attenuate eosinophilic airway inflammation when used as an adjunct to corticosteroid therapy in patients with mild asthma. ${ }^{84}$ Suplatast inhibits Th2-type cytokines, which are purportedly involved in the pathogenesis of asthma. Sano et al. compared outcomes in 15 patients with mild asthma treated with suplatast tosilate for 6 weeks and 13 control patients. The treatment group showed a significant improvement in the concentration of histamine following a challenge while the control patients did not. Similarly, significant improvements in peak expiratory flow and in symptom scores were observed in patients in the treatment group, but not in controls. Treatment with suplatast was associated with a reduction in average number of infiltrating eosinopils. The average number of CD4+ (clusters of differentiation) and CD25+ T cells also dropped with suplatast therapy. These outcomes were not observed in controls. ${ }^{85}$

\section{DNA Vaccination}

It has been proposed that one potentially effective strategy for modifying the asthma disease process is to use immunostimulatory DNA sequences to bias the immune system away from a Th2 response and toward a Th1 response. ${ }^{86}$ Using a mouse model, Horner et al. noted that airway and systemic eosinophilia were significantly suppressed following administration of a vaccination containing DNA sequences and noted a suppressed airway and systemic eosinophilia in response to an inhaled allergen. ${ }^{86}$ Inhibition of airway inflammation and eosinophilia has also been noted in mice using a vaccine that induces an immune response directed at IL-5. Immunotherapy with vaccinations appears to be effective in animal models, but extensive further research is required to demonstrate their applicability in humans.

In summary, biologic agents under development for the treatment of asthma offer the promise of therapies that target specific steps in the pathogenesis of the disease. However, with the exception of the IgE blocker omalizumab, these experimental therapies are several years away from potential use in regular clinical practice. Because of their experimental status, no data exist comparing the cost-effectiveness of these agents with more conventional therapies. As novel biologic therapies become available, the most appropriate use will need to be determined, particularly considering the current availability of many safe and effective pharmacotherapies that provide sufficient asthma control in the majority of patients.

\section{Conclusions}

Asthma care remains suboptimal despite major therapeutic advances over the past decade and the widespread dissemination 
of asthma management guidelines. Asthma is associated with significant morbidity and mortality, and, clearly, the primary goals of therapy are to decrease the number of exacerbations, reduce the severity of symptoms, reduce ED use and hospital admissions, and improve QOL. While progress has been made in reducing the rates of adverse outcomes of asthma, there remain unacceptably high rates of adverse outcomes, particularly in patients with poorly controlled moderate-to-severe disease. It is these patients who are suboptimally controlled on current standard-of-care medications and who consume a disproportionate percentage of the health care resources used in asthma care.

Patients with poorly controlled asthma stand to benefit from management strategies that guide clinicians and managed care organizations in making appropriate treatment decisions. Numerous observational studies have shown that practitioner compliance with national treatment guidelines and patient adherence to treatment regimens is less than optimal. Targeted educational initiatives for pharmacists and other health care professionals, as well as for patients, may help improve clinical and economic outcomes.

Asthma patients will also benefit from the continuing development of novel biologic therapies that specifically target the mechanisms responsible for persistent airway inflammation. The recently approved $\operatorname{IgE}$ blocker omalizumab and the numerous cytokine modulators and other biologically engineered agents still in development offer patients with poorly controlled, moderateto-severe asthma promising alternatives to currently available treatments. However, the cost-effectiveness of these agents has yet to be determined.

\section{ACKNOWLEDGMENT}

The research and editorial support of Keith Engelke is greatly appreciated.

\section{DISCLOSURES}

Funding for this research was provided by an unrestricted educational grant from Genentech Inc. and Novartis Pharmaceuticals Corporation, and was obtained by author William W. Storms. The author discloses participation in speakers' bureaus or paid consultant work for AstraZeneca, Aventis, Genetech, Merck, Novartis, and Schering-Plough and work as principal investigator in research funded by 3M, Adams Labs, Aerogen, AstraZeneca, Aventis, Bayer, Immunex, Ivax, Merck, Novartis, Pfizer, Schering-Plough, Sepracor, and others. Administrative and technical support for this study was provided by Keith Engelke, Dover Communications, Blue Bell, Pennsylvania.

\section{REFERENCES}

1. National Heart, Lung, and Blood Institute. National Asthma Education and Prevention Program Expert Panel. Expert Panel Report 2. Guidelines for the Diagnosis and Management of Asthma. Bethesda, MD: National Institutes of Health; April 1997:1-86. NIH Publication No 97-4051.

2. Mannino DM, Homa DM, Akinbami LJ, et al. Surveillance for asthma-United States, 1980-99. MMWR. 2002;51:1-13.

3. Smith DH, Malone DC, Lawson KA, et al. A national estimate of the economic costs of asthma. Am J Respir Crit Care Med. 1997;156:787-93.

4. Serra-Batlles J, Plaza V, Morejon E, et al. Costs of asthma according to degree of severity. Eur Respir J. 1998;(12):1322-26.
5. Godard P, Chanez P, Siraudin L, et al. Costs of asthma are correlated with severity: a 1-yr. prospective study. Eur Respir J. 2002;19:61-67.

6. National Institutes of Health and National Heart, Lung, and Blood Institute. Global Initiative for Asthma: Global Strategy for Asthma Management and Prevention. Bethesda, MD: National Institutes of Health; 2002. NIH Publication No. 02-3659.

7. National Committee on Quality Assurance. The State of Health Care Quality, 2002. Use of appropriate medications for people with asthma. Available at: http://www.ncqa.org/sohc_2002_asthma.html. Accessed August 16, 2003.

8. Fuhlbrigge AL, Adams RJ, Guilbert TW, et al. The burden of asthma in the United States. Am J Respir Crit Care Med. 2002;166:1044-49.

9. Asthma in America Survey. Executive Summary. Available at: http://www. asthmainamerica.com/execsum_over.htm. Accessed September 4, 2003.

10. National Center for Health Statistics. Asthma Prevalence, Health Care Use and Mortality, 2000-2001. 2003. Available at:

http://www.cdc.gov/nchs/products/pubs/pubd/hestats/asthma/asthma.htm. Accessed March 23, 2003.

11. National Institutes of Health. Data Fact Sheet. Asthma Statistics. 1999. Available at: http://www.nhlbi.nih.gov/health/prof/lung/asthma/asthstat.pdf. Accessed March 23, 2003.

12. Headrick L, Crain E, Evans D, et al. National asthma education and prevention program working group on the quality of asthma care. Am J Resp Crit Care Med. 1996;154(suppl):S96-S118.

13. Goodman D, Stukel T, Chang C. Trends in pediatric asthma hospitalization rates: regional and socioeconomic differences. Pediatrics. 1998;101:208-13.

14. American Lung Association. Trends in asthma morbidity and mortality, 2002. Available at: http://www.lungusa.org/data/lc/asthmach_1.html.

15. Weiss KB, Sullivan SD, Lyttle CS. Trends in the cost of illness for asthma in the United States, 1985-1994. J Allergy Clin Immunol. 2000;106:493-99.

16. Weiss KB, Gergen PJ, Hodgson TA. An economic evaluation of asthma in the United States. N Engl J Med. 1992;326:862-66.

17. Eisner MD, Ackerson LM, Chi F, et al. Health-related quality of life and future health care utilization for asthma. Ann Allergy Asthma Immunol. 2002;89:46-55.

18. Hoskins G, McCowan C, Neville RG, et al. Risk factors and costs associated with an asthma attack. Thorax. 2000;55:19-24.

19. Sullivan S, Crown B, Bresnahan B, et al. Health care utilization and expenditures: a study of moderate-to-severe asthma in the United States. Allergy Clin Immunol; 2003;109. In press.

20. Hayden M. High-level health care utilization in severe and difficult-to-treat asthma. J Allergy Clin Immunol. 2002;109:S293.

21. Sauni R, Oksa P, Vattulainen K, Uitti J, Palmroos P, Roto P. The effects of asthma on the quality of life and employment of construction workers. Occup Med (Lond). 2001;51:163-67.

22. Sawyer MG, Spurrier N, Whaites L, Kennedy D, Martin AJ, Baghurst P. The relationship between asthma severity, family functioning and the health-related quality of life of children with asthma. Qual Life Res. 2000;9:1105-115.

23. Sawyer MG, Spurrier N, Kennedy D, Martin J. The relationship between the quality of life of children with asthma and family functioning. J Asthma. 2001;38:279-84

24. Meyer IH, Sternfels P, Fagan JK, Ford JG. Asthma-related limitations in sexual functioning: an important but neglected area of quality of life. Am J Public Health. 2002;92:770-72.

25. Roghmann MC, Sexton M. Adherence to asthma guidelines in general practices. J Asthma. 1999;36:381-87.

26. Barr RG, Somers SC, Speizer FE, Camargo CA Jr. Patient factors and medication guideline adherence among older women with asthma. Arch Intern Med. 2002;162:1761-68 
27. Piecoro LT, Potoski M, Talbert JC, Doherty DE. Asthma prevalence, cost, and adherence with expert guidelines on the utilization of health care services and costs in a state Medicaid population. Health Serv Res. 2001;36:357-71.

28. Stoloff SW. Improving adherence to asthma therapy: what physicians can do. Am Fam Physician. 2000;61:2328, 2330, 2337.

29. Schmier JK, Leidy NK. The complexity of treatment adherence in adults with asthma: challenges and opportunities. J Asthma. 1998;35:455-72.

30. Apter AJ, Reisine ST, Affleck G, Barrows E, ZuWallack RL. Adherence with twice-daily dosing of inhaled steroids. Socioeconomic and health-belief differences. Am J Respir Crit Care Med. 1998;157:1810-17.

31. Kelloway JS, Wyatt RA, Adlis SA. Comparison of patients' compliance with prescribed oral and inhaled asthma medications. Arch Intern Med. 1994;154: 1349-52.

32. Crystal-Peters J, Neslusan C, Crown WH, Torres A. Treating allergic rhinitis in patients with comorbid asthma: the risk of asthma-related hospitalizations and emergency department visits. J Allergy Clin Immunol. 2002;109:57-62.

33. Ben-Noun L. Characteristics of comorbidity in adult asthma. Public Health Rev. 2001;29:49-61.

34. Wark PA, Gibson PG, Johnston SL. Exacerbations of asthma: addressing the triggers and treatments. Monaldi Arch Chest Dis. 2001;56:429-35.

35. Goodwin RD, Pine DS. Respiratory disease and panic attacks among adults in the United states. Chest. 2002;122:645-50.

36. Galil N. Depression and asthma in children. Curr Opin Pediatr. 2000;12:331-35.

37. Ernst P, Spitzer WO, Suissa S, et al. Risk of fatal and near-fatal asthma in relation to inhaled corticosteroid use. JAMA. 1992;268:3462-64.

38. National Heart Lung, and Blood Institute. National Asthma Education and Prevention Program. Expert Panel Report: Guidelines for the diagnosis and management of asthma-Update on selected topics 2002. J Allergy Clin Immunol. 2002;110(suppl):S141-S183.

39. Doerschug KC, Peterson MW, Dayton CS, Kline JN. Asthma guidelines: an assessment of physician's understanding and practice. Am J Crit Care Med. 1999;159:1735-41

40. Donahue JG, Fuhlbrigge AL, Finkelstein JA, et al. Asthma pharmacotherapy and utilization by children in 3 managed care organizations. J Allergy Clin Immunol. 2000;106:1108-14.

41. Lang DM, Sherman MS, Polansky M. Guidelines and realities of asthma management. The Philadelphia story. Arch Intern Med. 1997;157:1193-2000.

42. Legorreta AP, Christian-Herman J, O'Connor RD, Hasan MM, Evans R, Leung KM. Compliance with national asthma management guidelines and specialty care: a health maintenance organization experience. Arch Intern Med. 1998;158 457-64.

43. Flores G, Lee M, Kastner B, Bauchner H. Pediatricians' attitudes, beliefs, and practices regarding clinical practice guidelines: a national survey. Pediatrics. 2000; 105:496-501.

44. Christakis DA, Rivara FP. Pediatricians' awareness of and attitudes about four clinical practice guidelines. Pediatrics. 1998;101:825-30.

45. Picken HA, Greenfield S, Teres D, Hirway PS, Landis JN. Effect of local standards on the implementation of national guidelines for asthma. J Gen Intern Med. 1998;13:659-63.

46. Homer CJ. Asthma disease management. N Engl J Med. 1997;337:1461-63. 47. Stavish S. Managed care fumbles asthma guidelines. Pediatric News. March 1998:38.

48. Knoell DL, Pierson JF, Marsh CB, Allen JN, Pthak DS. Measurement of outcomes in adults receiving pharmaceutical care in a comprehensive asthma outpatient clinic. Pharmacotherapy. 1998;18:1365-74.

49. Fischer LR, Scott LM, Boonstra DM, et al. Pharmaceutical care for patients with chronic conditions. J Am Pharm Assoc (Wash). 2000;40:174-80.
50. Narhi U, Airaksinen M, Tanskanen P, Erlund H. Therapuetic outcomes monitoring by community pharmacists for improving clinical outcomes in asthma. J Clin Pharm Ther. 2000;25:177-83.

51. Yanchick JK. Implementation of a drug therapy monitoring clinic in a primary care setting. Am J Health Syst Pharm. 2000;57(suppl 4):S30-S34.

52. Weinberger M, Murray MD, Marrero DG, et al. Effectiveness of pharmacist care for patients with reactive airway disease: a randomized controlled trial. JAMA. 2002;288:1594-602.

53. Diette GB, Skinner EA, Nguyen TT, Markson L, Clark BD, Wu AW. Comparison of quality of care by specialist and generalist physicians as usual source of asthma care for children. Pediatrics. 2001;108:432-37.

54. Storms B, Olden L, Nathan R, Bodman S. Effect of allergy specialist care on the quality of life in patients with asthma. Ann Allergy Asthma Immunol. 1995; 75:491-94

55. Zeiger RS, Heller S, Mellon MH, Wald J, Falkoff R, Schatz M. Facilitated referral to asthma specialist reduces relapses in asthma emergency room visits. J Allergy Clin Immunol. 1991;87:1160-68.

56. Westley CR, Spiecher B, Starr L, et al. Cost effectiveness of an allergy consultation in the management of asthma. Allergy Asthma Proc. 1997;18:15-18.

57. Wu AW, Young Y, Skinner EA, et al. Quality of care and outcomes of adults with asthma treated by specialists and generalists in managed care. Arch Int Med. 2001;161:2554-60

58. Frieri M, Therattil J, Dellavecchhia D, Rockitter S, Pettit J, Zitt M. A preliminary retrospective treatment and pharmacoeconomic analysis of asthma care provided by allergists, immunologists, and primary care physicians in a teaching hospital. J Asthma. 2002;39:405-12.

59. Milgrom H, Bender B, Sarlin N, Leung D. Difficult-to-control asthma: the challenge posed by non-compliance. Am J Asthma Allergy Pediatr. 1994;3:141-46.

60. Barr RG, Somers SC, Speizer FE, Camargo CA Jr. Patient factors and medication guideline adherence among older women with asthma. Arch Intern Med. 2002;162:1761-68.

61. Stoloff SW, Janson S. Providing asthma education in primary care practice. Am Fam Physician. 1997;56:117-126, 131-134, 142.

62. Apter AJ, Reisine ST, Affleck G, Barrows E, ZuWallack RL. Adherence with twice-daily dosing of inhaled steroids. Socioeconomic and health-belief differences. Am J Respir Crit Care Med. 1998;157:1810-17.

63. Schmaling KB, Afari N, Blume AW. Predictors of treatment adherence among asthma patients in the emergency department. J Asthma. 1998;35:631-36.

64. Bender B, Milgrom H, Rand C. Nonadherence in asthmatic patients: is there a solution to the problem? Ann Allergy Asthma Immunol. 1997;79:177-85.

65. Milgrom H, Bender B, Ackerson L, Bowry P, Smith B, Rand C. Noncompliance and treatment failure in children with asthma. J Allergy Clin Immunol. 1996;98:1051-57.

66. Suissa S, Ernst P, Benayoun S, Baltzan M, Cai B. Low-dose inhaled corticosteroids and the prevention of death from asthma. N Engl J Med. 2000;343:33236.

67. Suissa S, Ernst P, Kezouh A. Regular use of inhaled corticosteroids and the long-term prevention of hospitalization for asthma. Thorax. 2002;57:880-84

68. Bender B, Wamboldt FS, O'Connor SL, et al. Measurement of children's asthma medication adherence by self report, mother report, canister weight, and Doser CT. Ann Allergy Asthma Immunol. 2000: 85:416-21.

69. Trautner C, Richter B, Berger M. Cost-effectiveness of a structured treatment and teaching programme on asthma. Eur Respir J. 1993;6:1485-91.

70. Cochrane GM. Compliance and outcomes in patients with asthma. Drugs. 1996;52(suppl):12-19.

71. Partridge MR. Delivering optimal care to the person with asthma: what are the key components and what do we mean by patient education? Eur Respir J. 1995;8:298-305. 
72. Hunt LW. How to manage difficult asthma cases. An action plan for physicians and patients. Postgrad Med. 2001;109:61-68.

73. Omalizumab [prescribing information]. San Francisco, CA: Genentech, Inc., and East Hanover, NJ: Novartis Pharmaceuticals Corporation; 2003. Available at: http://www.gene.com/gene/common/inc/pi/xolair.jsp. Accessed August 15, 2003

74. Busse W, Corren J, Lanier BQ, et al. Omalizumab, anti-IgE recombinant humanized monoclonal antibody, for the treatment of severe allergic asthma. J Allergy Clin Immunol. 2001;108:184-90.

75. Soler M, Matz J, Townley R, Buhl R, et al. The anti-IgE antibody omalizumab reduces exacerbations and steroid requirement in allergic asthmatics. Eur Respir J 2001;18:254-61.

76. Barnes PJ. Cytokine modulators as novel therapies for asthma. Ann Rev Pharmacol Toxicol. 2002;42:81-98

77. Barnes PJ, Chung KF, Page CP. Inflammatory mediators of asthma: an update Pharm Rev. 1998;50(4):515-96.

78. Borish LC, Nelson HS, Lanz MJ, et al. Interleukin-4 receptor in moderate atopic asthma: a phase I/II randomized, placebo-controlled trial. Am J Respir Crit Care Med. 1999; 160:1816-23.

79. Leckie MJ, ten Brinke A, Khan J, et al. Effects of an interleukin-5 blocking monoclonal antibody on eosinophils, airway hyperresponsiveness, and the late asthmatic response. Lancet. 2000;356:2144-48.
80. O'Byrne PM, Inman MD, Parameswaran K. The trials and tribulations of IL-5, eosinophils, and allergic asthma. J Allergy Clin Immunol. 2001;108:503-08

81. Tomita K, Lim S, Hanazawa T, et al. Attenutated production of IL-10 and IL-12 in monocytes from patients with severe asthma. Clin Immunol. 2002; 102:258-66.

82. Lim S, Crawley E, Woo P, Barnes PJ. Haplotype associated with low interleukin-10 production in patients with severe asthma. Lancet. 1998;352:113.

83. Bryan SA, O'Connor BJ, Matti S, et al. Effects of recombinant human interleukin-12 on eosinophils,airway hyper-responsiveness, and the late asthmatic response. Lancet. 2000;356:2149-53.

84. Tamaoki J, Kondo M, Sakai N, et al. Effect of suplatast tosilate, a Th2 cytokine inhibitor, on steroid-dependent asthma: a double-blind randomised study. Lancet. 2000;356:273-78.

85. Sano Y, Suzuki N, Yamada H, et al. Effects of suplatast tosilate on allergic eosinophilic airway inflammation in patients with mild asthma. J Allergy Clin Immunol. 2003;111:958-66.

86. Horner AA,Van Uden JH, Zubeldia JM, et al. DNA-based immunotherapeutics for the treatment of allergic disease. Immunol Rev. 2001;179:102-18. 